\title{
Efficiency of coordinator-based osteoporosis intervention in fragility fracture patients: a prospective randomized trial
}

\author{
M. Osaki ${ }^{1}$ (D) R. Okuda ${ }^{2} \cdot$ Y. Saeki $^{3} \cdot$ T. Okano $^{4} \cdot \mathrm{K}$ Tsuda $^{5} \cdot$ T. Nakamura $^{6} \cdot$ Y. Morio $^{7} \cdot$ H. Nagashima $^{8} \cdot$ H. Hagino $^{1,2}$
}

Received: 11 October 2020 / Accepted: 4 January 2021 / Published online: 23 January 2021

(C) The Author(s) 2021

\begin{abstract}
Summary We examined the effectiveness of coordinators' interventions to prevent secondary fractures in patients with fragility fractures. These coordinator-based interventions improved bone density assessment implementation and treatment rates, and enhanced treatment persistence rates in the early stages following fractures.

Introduction This study aimed to determine the efficiency of coordinator-based osteoporosis intervention in fragility fracture patients during a 2-year period.

Methods A prospective intervention randomized control study was conducted at seven medical facilities from January 2015 to March 2017. Postmenopausal women and men over 50 years old with fragility fractures were randomly divided into the coordinator intervention (LI; 70 patients) and without intervention (non-LI; 71 patients) groups. The osteoporosis treatment rate, osteoporosis treatment persistence rate, fall rate, fracture incidence rate, and bone density measurement rate 3 months, 6 months, 1 year, and 2 years after registration were compared between the two groups. Non-parametric tests were used to analyze data at each inspection period. Results The osteoporosis treatment initiation rate was significantly higher in the LI group than in the non-LI group $(85.7 \%$ vs. $71.8 \% ; p$ $=0.04)$. The LI group had significantly higher bone density assessment implementation rates than the non-LI group at the time of registration $(90.0 \%$ vs. $69.0 \% ; p=0.00)$ and 6 months after registration $(50.0 \%$ vs. $29.6 \% ; p=0.01)$, but not 1 or 2 years after registration. In addition, no significant differences in fall or fracture incidence rates were found between the two groups.

Conclusion The coordinator-based interventions for fragility fractures improved bone density assessment implementation and treatment rates and enhanced treatment persistence rates in the early stages following bone fractures. The findings suggest that liaison intervention may help both fracture and osteoporosis physicians for the evaluation of osteoporosis and initiation and continuation of osteoporosis medication.
\end{abstract}

Keywords Anti-osteoporosis treatment $\cdot$ Bone density assessment $\cdot$ Fall rate $\cdot$ Fracture liaison service $\cdot$ Secondary fracture prevention

\section{Introduction}

Osteoporosis is defined as a skeletal disorder characterized by compromized bone strength predisposing a person to an increased risk of fracture" [1]. Osteoporosis increases the

M. Osaki

osakim@tottori-u.ac.jp

1 Rehabilitation Division, Tottori University Hospital, Tottori 683-8504, Japan

2 School of Health Science, Faculty of Medicine, Tottori University, Tottori, Japan

3 Orthopedic Surgery Hospital Ward, Tottori University Hospital, Tottori, Japan risk of fracture, but the presence of decreased bone mass alone does not always result in symptoms and is often first known with the onset of fracture; thus, osteoporosis is referred to as a "silent disease." Fragility fractures caused by osteoporosis are well-known to increase the risk of further fragility fractures;

4 Department of Orthopedic Surgery, San-in Rosai Hospital, Tottori, Japan

5 Department of Orthopedic Surgery, Saiseikai Sakaiminato General Hospital, Tottori, Japan

6 Department of Orthopedic Surgery, Hakuai Hospital, Tottori, Japan

7 Department of Orthopedic Surgery, Misasa Onsen Hospital, Tottori, Japan

8 Department of Orthopedic Surgery, Tottori University, Tottori, Japan 
medical history of the wrist, vertebral body, or hip fractures increases the risk of further fractures in the wrist, vertebral body, and hip [2]. Fragility fractures induce lower activities of daily living (ADL), and repeated fractures further reduce mobility. For instance, a large-scale investigation involving 10,992 patients with hipfractures treated at 158 national facilities [3] showed that $87 \%$ of patients had independent ADL prior to fracture, but the rate decreased to $50 \%$ after 1 year following the fracture. In addition, studies found that women who experienced at least one vertebral body fracture had a higher mortality rate than women without vertebral body fractures [4]; that the 1-year survival rate of patients with hip fractures was lower than that of the general population [5]; and that among patients with hip fractures in nursing homes, $45 \%$ died and only $35 \%$ were able to walk 12 months after injury [6]. These findings suggest that fragility fractures are associated with decreased survival in the elderly.

There has been tremendous progress in osteoporosis treatment drugs in recent years, and these drugs suppress secondary fracture in over $50 \%$ of patients with fragility fracture; however, there have been few cases where suitable osteoporosis treatment was conducted in patients following fragility fracture. For instance, an investigation in Denmark [7] showed that the osteoporosis treatment rates following fracture were $16.5 \%$ and $39.6 \%$ in men and women, respectively, which are relatively low; in addition, there was an unfavorable persistence rate as well. Studies in Japan indicated that the osteoporosis treatment rate following hip fracture was low (less than 20\%) $[8,9]$. These findings indicate that fragility fractures are terrifying illnesses, and their onset induces further fracture, rapidly decreases ADL/quality of life (QOL), and even worsens vital prognosis. However, to date, no efficient re-fracturing prevention treatment is available.

In recent years, there has been more widespread use of fracture liaison service (FLS), which involves the intervention of coordinators (liaisons) who efficiently implement fragility fracture prevention through "multidisciplinary coordination" [10]. This secondary fracture prevention program involves multidisciplinary patient education, osteoporosis treatment commencement, exercise guidance, and fall prevention guidance for patients with fragility fractures. Secondary fracture prevention is conducted during the fracture treatment period at medical facilities and is thus easy to implement. Furthermore, the pain and lifestyle function limitations accompanying fracture are personally experienced, and it is thus easy to gain comprehension of osteoporosis treatment for the purposes of fracture prevention [11]. Therefore, FLS is considered to have superior treatment efficiency than primary fracture prevention [10].

There have been several secondary fracture prevention initiatives for Asian populations [12]. An authorization system for the education of medical staff with knowledge of osteoporosis and coordinators involved in secondary fracture prevention has recently started in Japan since 2014 [13]. However, no prospective studies have examined the effectiveness of coordinated secondary fracture prevention among Japanese populations. Accordingly, the objective of this study was to determine the effectiveness of liaisons who received coordinator authorization for osteoporosis treatment following the onset of fragility fracture in improvements of osteoporosis treatment and persistence rates and outcomes of secondary fracture prevention.

\section{Methods}

\section{Study design}

This study was a prospective, randomized, comparative open study with 2-year intervention. The primary endpoints were the osteoporosis treatment commencement and persistence rates. The secondary endpoints were falling incidence rate and fracture incidence rate.

\section{Patients}

Patients with fragility fractures within 3 months of an injury, who were injured between January 2015 and March 2017, and treated at one of the seven medical facilities in Tottori Prefecture, were included in the study (These seven hospitals accept fragility fracture patients and perform fracture surgery). A fragility fracture was defined as a "non-traumatic fracture that occurred due to slight external force." Selected patients included postmenopausal women and men aged 50 years or older; they provided written consent following sufficient explanation and comprehension of research participation. Patients, whose investigation was difficult due to dementia, with fractures due to high-energy trauma, such as traffic/ falling accidents, and whose treatment and investigation were difficult due to severe complications, were excluded from the study. Fracture locations included the spine, hip, ribs, pelvis, proximal humerus, distal radius, and lower leg bones. Since the aim of the study was to investigate the effect of an intervention, not including all cases of fragility fracture included in this study. This was due to the limit number and burden on the liaison nurses, of whom there were only one or two in each facility. At the start of the study, the target number of patients was set at 150 .

\section{Setting}

Before this study, we independently created a pamphlet for refracturing prevention. The pamphlet could record osteoporosis treatment history, bone density inspection, falling and 
included information on exercise, daily lifestyle guidance, and dietary guidance for preventing re-fracturing. The number of coordinators included 13 in seven facilities. The coordinators earned their certification from the Japanese Osteoporosis Society, and the certification requirements are based on lecture attendance for a certain number of hours and passing an exam. They also regularly attend lectures to renew their manager certification [13]. In addition, study and information sessions were held every 4 months for this project to ensure that the manager's activities were occurring.

When patients with fragility fractures either sought a diagnosis at the outpatient care or were hospitalized at one of the participating research facilities, a liaison checked the chart information and picked up those who conformed to the eligibility criteria and did not meet exclusion criteria. Patients with (1) hip fracture, (2) vertebral body fracture, or (3) other fractures were randomly and dynamically allocated based on the fracture type into coordinator intervention (liaison intervention; LI) and without intervention (non-liaison intervention; non-LI) groups using an enveloped method. A previously assigned facility registration number and an identification (ID) number created at each facility were then recorded, and a registration sheet was entered for all patients; all information was then sent to a registration/data center. The allocation results and research subject list were managed by only the liaison at each facility, and both physicians and patients were blinded to the allocation results. Baseline observation endpoints included sex, age at the time of registration, diagnosis date, walking state prior to injury/onset, osteoporosis treatment presence, types of osteoporosis treatment, fracture date, fracture location and type, injury location, reason for injury, medical history other than fracture, and history of fracture.

Standard treatments, such as osteosynthesis for fracture of the long bones and corset treatment for spinal vertebral fractures, were performed according to the hospital policy in both groups. None of the treatments were fixed in the seven hospitals. The liaison requested bone density assessments and osteoporosis drug treatment to the physicians for the LI group at the time of research registration. Fracture risk assessment using fracture risk assessment tool (FRAX), EuroQOL 5 dimensions 5-level (EQ-5D-5L)-based QOL assessments, fall prevention/lifestyle guidance using created pamphlets, and osteoporosis treatment education were conducted by the liaison. Only the created pamphlets were distributed, and FRAXbased fracture risk assessment and QOL assessment were conducted for the non-LI group. The dynamic allocation was conducted for both groups, and assessments were conducted for drug administration persistence, osteoporosis treatment drug administration, bone density assessment implementation, fall/fracture/complication onset presence, and health inspections (EQ-5D-5L) at the time of registration, as well as 3 months, 6 months, 1 year, and 2 years after registration (henceforth, "after 3 months," "after 6 months," "after 1 year," and "after 2 years," respectively). Diagnoses were encouraged at each time period, guidance was implemented through the interview for the LI group, and patients who could not conduct diagnoses had their assessments by phone. The non-LI group underwent the survey and questionnaire by mail only.

The primary endpoints were the osteoporosis treatment commencement rate and the osteoporosis treatment persistence rate. As for the drug administration persistence rate, persistence among those with prescriptions from treatment institutions was classified as never forgetting to take their administered prescriptions or only missing 1 or 2 administrations. As for patients who used injections, the liaison at each facility confirmed from the medical facility whether the patient was persistently administered the drug, and persistence was defined as persistent administration of the drug.

The secondary endpoints were fall and fracture rates.

Falls were defined according to the definition provided by Gibson, which was published by the Kellogg International Work Group [14], as "unintentionally coming to the ground or to a lower level." Falling incidents were recorded in a "bone health notebook" distributed to patients, and records were confirmed either during diagnosis or during interviews with liaisons. The fall incidence rate was assessed based on falls that occurred even once during the observation period, and the percentages of fall presence were compared between the LI and non-LI groups. The bone fracture incidence rate was calculated based on clinical fractures that were confirmed by Xray inspection, $\mathrm{CT}$ inspection, and MRI inspection during symptomatic periods or at the time of falling. The frequencies of fall incidents were compared between the LI and non-LI groups. Furthermore, bome mineral density were performed at registration and at 6 months, 1 year, and 2 years after registration. We compared the percentages of bone mineral density assessments between the LI and non-LI groups. And the incidences of complications (pneumonia, dementia, or bedsores) at the time of registration were reported by the patient or family members, and the differences in incidence at each observation period between the LI and non-LI groups were analyzed.

\section{Statistical analyses}

The efficacy analysis used the per-protocol set, and the analysis of adverse events used the full analysis set. Statistical analyses for assessment categories at each inspection period used non-parametric tests (chi-squared test or Fisher's exact test). As for the secondary fracture incidence rate, the Kaplan-Meier estimator with secondary fracture incidence as an endpoint was used to compare the LI and non-LI groups, and the cumulative survival rate at the 2-year mark was analyzed using the log-rank test. The significance level was set at $p<0.05$. 
All statistical analyses were contracted to the Institute of Statistical Mathematics of the Research Organization of Information and Statistics, and assessments were independently conducted. Data analyses were performed using SAS/STAT software, version 9.4 (SAS Institute Inc., Cary, NC, USA).

\section{Ethical considerations}

All patients at the participating research facilities were assigned previously allocated facility registration numbers and patient IDs, patient information was recorded in a registration sheet using a research subject authorization code, and all individual information was removed from the registration sheet for central management.

\section{Results}

\section{Patient characteristics}

From January 2015 to March 2017, the total number of postmenopausal women and men over 50 years of age with fractures treated at the seven medical facilities in Tottori Prefecture were 5907. The different fracture types included 1326 hip fractures, 2111 spine fractures, and 2470 other site fractures. A total of 163 patients were registrated, of which 163 were deemed suitable for the study, provided consent, and did not meet the exclusion criteria (Fig. 1). From the randomized 163 patients, 83 were allocated to the LI group (16 men, 67 women) and 80 to the non-LI group (12 men, 68 women), with no significant difference in the ratio of men to women between the two groups $(p=0.469)$. The mean age was 76.2 years in the LI group and 76.8 years in the non-LI group, which was not statistically significant $(p=0.628)$. After registration, several patients withdrew consent, died, met exclusion criteria, or were lost to the early follow-up. The final number of investigated and tracked patients was 70 for the LI group and 71 for the non-LI group (Table 1). The average age of patients who were tracked for 2 years was $76.3 \pm 8.6$ years in the LI group and $76.5 \pm 7.8$ years in the non-LI group. There were 12 men and 58 women in the LI group and 11 men and 60 women in the non-LI group. No significant differences in age and sex were found between the two groups. The number of patients with a positive history of fragility fracture was similar between the groups: 21 in LI group $(n=70)$ and 22 in non-LI group $(n=71)(p=0.811)$. The fracture locations in patients were as follows: vertebral body, 93 patients (66.0\%); hip, 18 patients (12.8\%); distal radius and proximal humerus, 19 patients (13.5\%); and others, 11 patients $(7.8 \%)$. Fractures were observed in the hip, vertebral body, proximal humerus/distal radius, and other locations in $9,46,9$, and 6 patients, respectively, in the LI group and $9,47,10$, and 5 patients, respectively, in the non-LI group; no significant differences were noted between the two groups. Due to the small number of male patients registrated in this study, sex differences could not be assessed.

\section{Anti-osteoporosis treatment}

A total of 21 patients (30.0\%) in the LI group and 21 patients $(29.6 \%)$ in the non-LI group underwent osteoporosis treatment prior to registration; approximately one-third of patients in both groups were diagnosed with osteoporosis, and no significant differences were observed between the two groups (Table 2).

The osteoporosis treatment commencement rate (one primary endpoint) was significantly higher in the LI group $(85.7 \%)$ than in the non-LI group $(71.8 \%)(p=0.04)$. The osteoporosis treatment drug persistence rates (the other primary endpoint) were $92.9 \%$ in the LI group and $81.7 \%$ in the non-LI group after 3 months, $87.1 \%$ in the LI group and $78.9 \%$ in the non-LI group after 6 months, and $87.1 \%$ in the LI group and $78.9 \%$ in the non-LI group after 1 year; although the LI group had higher osteoporosis treatment drug persistence rates than the non-LI group during all periods, no significant differences were observed between the two groups. Furthermore, investigations on forgetting to take the administered drugs were conducted with classifications including "none," "occasionally," "frequently," and "not administering drug." The "occasionally" forgetting was the most common at any given period, and no significant differences were observed between the two groups.

\section{Bone density measurement}

The bone density assessment implementation rates were significantly higher in the LI group than in the non-LI group at the time of registration $(90.0 \%$ vs. $69.0 \% ; p=0.00)$ and after 6 months $(50.0 \%$ vs. $29.6 \% ; p=0.01)$, but not after $1(61.4 \%$ vs. $52.1 \% ; p=0.26)$ or $2(54.3 \%$ vs. $63.4 \% ; p=0.32)$ years (Table 3). The implementation rate gradually decreased in the LI group but increased in the non-LI group during the test period. Due to the large number of patients who were assessed and treated for osteoporosis, there was no difference between the facilities. However, there was no significant difference in the number of patients registrated at each facility. The results of bone density assessment showed that 41 patients in the LI group had a young adult mean $(\mathrm{YAM})<70 \%$ (T-score $<-2.5$ SD), 15 patients had a YAM of 70-80\% (T-score -2.5 SD-1.7 SD), and seven patients had a YAM $>80 \%$ (T-score $>-$ 1.7 SD). In the non-LI group, 28 patients had a $\mathrm{YAM}<70 \%$, 
Fig. 1 Flow chart of patient selection. LI, coordinator intervention group; non-LI, without intervention group

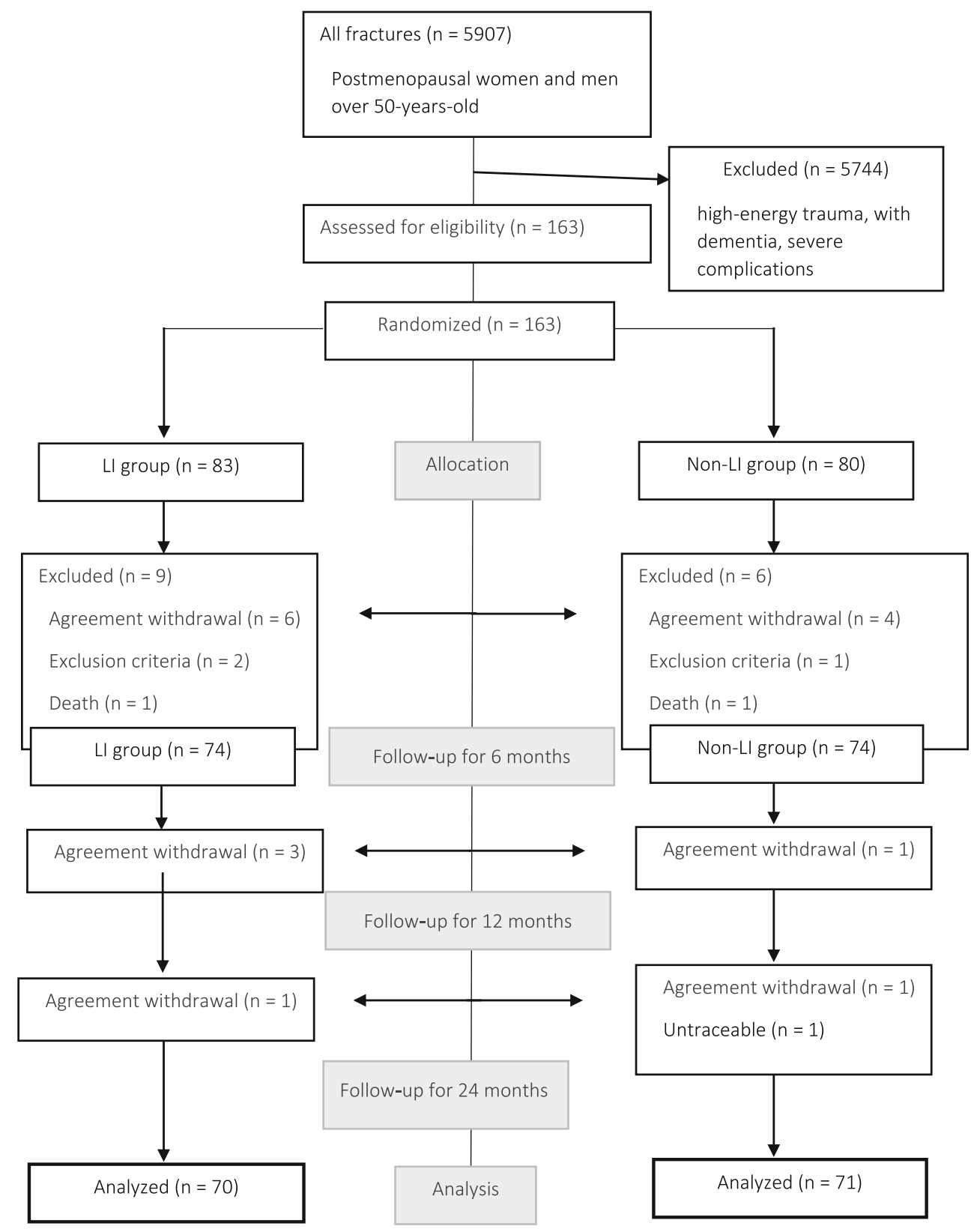

and 7 patients with repeated falls in the LI and non-LI groups, respectively.

\section{Fracture rate}

No significant differences in the fracture rates were observed between the LI and non-LI groups after 3 months $(1.5 \%$ vs. $4.3 \%), 6$ months $(1.5 \%$ vs. $3.0 \%), 1$ year $(4.5 \%$ vs. $8.8 \%)$, and 2 years $(8.6 \%$ vs. $7.5 \%)$ (Table 4). Moreover, no significant differences in the survival rate, which used fracture as an endpoint, were observed between the LI and non-LI groups $(86.1 \%$ vs. $81.0 \%)$. 
Table 1 Baseline patient characteristics

\begin{tabular}{llll}
\hline & LI $(n=70)$ & Non-LI $(n=71)$ & $p$ values \\
\hline Age (years) & $76.3 \pm 8.6$ & $76.5 \pm 7.8$ & 0.876 \\
Sex $(n / \%)$ & & & \\
Men & $12(17.1)$ & $11(15.5)$ & 0.791 \\
Women & $58(82.9)$ & $60(84.5)$ & 0.791 \\
Past fragility fracture $(n / \%)$ & $21(30.0)$ & $22(31.0)$ & 0.811 \\
History $(n / \%)$ & & & 0.986 \\
Hypertension & $31(44.3)$ & $32(45.1)$ & 0.832 \\
Diabetes & $9(12.9)$ & $10(14.1)$ & 0.393 \\
Cerebral infarction sequelae & $4(5.7)$ & $2(2.8)$ & 0.517 \\
Heart disease & $10(14.3)$ & $13(18.3)$ & 0.985 \\
Rheumatoid arthritis & $3(4.3)$ & $3(4.2)$ & 0.312 \\
Hyperthyroidism & $1(1.4)$ & $0(0.0)$ & 0.186 \\
Other disease & $14(20.0)$ & $21(29.6)$ & \\
Fracture sites $(n / \%)$ & & & 0.974 \\
Hip & $9(12.9)$ & $9(12.7)$ & 0.952 \\
Vertebral body & $46(65.7)$ & $47(66.2)$ & 0.831 \\
Distal radius/proximal humerus & $9(12.9)$ & $10(14.1)$ & 0.735 \\
Other sites & $6(8.6)$ & $5(7.0)$ & \\
\hline
\end{tabular}

$L I$, coordinator intervention group; non- $L I$, without intervention group. Data were analyzed using chi-squared test or Fisher's exact test $(p<0.05)$

\section{Adverse events}

There was no onset of anti-resorptive agents-related osteonecrosis of the jaw or atypical fracture during the follow-up observation period. Furthermore, there were no differences in other adverse events between the LI and non-LI groups.

\section{Discussion}

This study found that the treatment rate for osteoporosis was high in the LI group at all observation points, especially at the time of study registration. In detail, the LI group had

Table 2 Anti-osteoporotic treatment received

\begin{tabular}{llll}
\hline Time & LI $(n=70)$ & Non-LI $(n=71)$ & $p$ values \\
\hline Before registration & $21(30.0 \%)$ & $21(29.6 \%)$ & 1.00 \\
Baseline & $60(85.7 \%)$ & $51(71.8 \%)$ & $0.04^{*}$ \\
3 months & $65(92.9 \%)$ & $58(81.7 \%)$ & 0.78 \\
6 months & $61(87.1 \%)$ & $56(78.9 \%)$ & 0.37 \\
1 year & $61(87.1 \%)$ & $56(78.9 \%)$ & 0.37 \\
2 years & $52(74.6 \%)$ & $54(77.5 \%)$ & 0.84 \\
\hline
\end{tabular}

$L I$, coordinator intervention group; non- $L I$, without intervention group. Data were analyzed using chi-squared test or Fisher's exact test $(* p<0.05)$ significantly higher osteoporosis treatment rates at the time of registration in the LI group than in the non-LI group $(85.7 \%$ vs. $71.8 \%)$, but there were no significant differences in osteoporosis treatment rates between the two groups after 3 months. In addition, the bone density assessment implementation rates were significantly higher in the LI group than in the non-LI group at the time of registration $(90.0 \%$ vs. $69.0 \%)$ and after 6 months $(50.0 \%$ vs. $29.6 \%$ ) but was not significantly different between the two groups after 1 year. Moreover, the fall and fracture rates had no significant differences between the two groups throughout the testing period.

The FLS, which was commenced in the late 1990s in the UK $[10,15]$, is a system in which coordinators (liaisons) efficiently implement fragility fracture prevention through "multidisciplinary coordination" [10]. However, the commencement rate of osteoporosis drug administration for secondary

Table 3 Bone mineral measurements performed

\begin{tabular}{llll}
\hline Time & LI $(n=70)$ & Non-LI $(n=71)$ & $p$ values \\
\hline Baseline & $63(90.0 \%)$ & $49(69.0 \%)$ & $0.00^{*}$ \\
6 months & $35(50.0 \%)$ & $21(29.6 \%)$ & $0.01^{*}$ \\
1 year & $43(61.4 \%)$ & $37(52.1 \%)$ & 0.26 \\
2 years & $38(54.3 \%)$ & $45(63.4 \%)$ & 0.32 \\
\hline
\end{tabular}

$L I$, coordinator intervention group; non- $L I$, without intervention group. Data were analyzed using chi-squared test or Fisher's exact test $(* p<0.05)$ 
Table 4 Fall and fracture rates

\begin{tabular}{llllll}
\hline Time & Fall & & & Fracture & \\
\cline { 2 - 3 } \cline { 5 - 6 } \cline { 5 - 6 } & LI & Non-LI & LI & Non-LI \\
\hline 3 months & $2 / 65(3.0 \%)$ & $7 / 65(10.8 \%)$ & $1 / 68(1.5 \%)$ & $3 / 69(4.3 \%)$ \\
6 months & $7 / 66(10.6 \%)$ & $8 / 67(11.9 \%)$ & & $1 / 67(1.5 \%)$ & $2 / 67(3.0 \%)$ \\
1 year & $10 / 66(15.2 \%)$ & $9 / 66(13.6 \%)$ & & $3 / 66(4.5 \%)$ & $6 / 68(8.8 \%)$ \\
2 years & $17 / 56(30.4 \%)$ & $18 / 62(29.0 \%)$ & & $5 / 58(8.6 \%)$ & $5 / 67(7.5 \%)$ \\
\hline
\end{tabular}

$L I$, coordinator intervention group; non- $L I$, without intervention group. Data were analyzed using chi-squared test and Fisher's exact test (not significant)

fracture prevention following fracture treatment is low in patients with fragility fractures, probably due to the low awareness of osteoporosis treatment among orthopedic surgeons who conduct surgical fracture treatment, as well as the fact that physicians who oversee fracture treatment and osteoporosis treatment are different persons. In addition, reported effects of the liaison service include higher osteoporosis treatment commencement rates [11]; higher treatment persistence rates $[16,17]$; and decreased medical costs due to the lower incidence of hip fracture and secondary fracture prevention, such as vertebral body fracture [10-12, 18, 19]. Furthermore, FLS-based interventions have been reported to not only reduce the fracture incidence rate but also improve the survival rate [18]. These clinical results indicate that the liaison service is an extremely effective initiative that suppresses fragility fractures; improves the ADL, QOL, and vital prognosis of patients; and results in reduced medical fees.

This study assessed liaison-based interventions with randomized controlled trials. The osteoporosis treatment commencement rates, one of the primary endpoints, were significantly higher in the LI group than in the non-LI group $(85.7 \%$ vs. $71.8 \% ; p=0.04]$ ). One study that examined FLS-based interventions showed that the osteoporosis treatment rate 6 months following fracture was $51.5 \%$ in the intervention group and 5.9\% in the non-intervention group [20], and another study reported the osteoporosis treatment rates of $42 \%$ in the intervention group and $19 \%$ in the non-intervention group [16]; in addition, the treatment rates after 1 year among 724 patients with hip fractures were $74.9 \%$ in the LI group and $12.3 \%$ in the non-LI group [21]. Notably, even minimal interventions were found to improve the treatment rates (from 12.6 to $31.2 \%$ ) [22]. The present study found extremely high drug treatment rates, even in the non-LI group, with $71.8 \%$ at the time of registration, $78.9 \%$ after 1 year, and $77.1 \%$ after 2 years, and there were no significant differences in drug treatment rates between the LI and non-LI groups after 3 months. Several possible explanations are as follows: the research manager, fracture physician, and osteoporosis treatment physician were the same person; liaison activity was not blinded to treatment physicians; osteoporosis treatment persisted; and investigations might trigger the patient's visit to the hospital. In addition, extremely high treatment rates were maintained because patients and physicians both were aware of liaison activity, which was thought to be effective in osteoporosis treatment and assessment $[23,24]$. The assignment of patients to the groups was randomized and the study was conducted with the treating physicians blinded, but the liaison activity was an open trial. However, the liaisons proceeded with the examination and treatment, which was overseen by the treating physician. The majority of previous studies that showed significant effects of coordinator intervention were cluster-controlled trials or historical control trials, and largescale randomized control trials [25] showed no significant differences in the treatment rates. It has been considered that there are limitations to assessing the effects of coordinator intervention in randomized trials at the same facility.

Studies on bone density assessments after fracture indicated that liaison intervention resulted in improvements from 7.9 to $39.6 \%$ [22], and an assessment 6 months after fracture identified an improvement rate of $51.5 \%$ in the intervention group and $5.9 \%$ in the non-intervention group [20]. The bone density assessment implementation rates in the present study were significantly higher in the LI group than in the non-LI group at the time of registration $(90.0 \%$ vs. $69.0 \% ; p=0.00)$ and after 6 months $(50.0 \%$ vs. $29.6 \% ; p=0.01)$. The extremely high assessment rates and osteoporosis treatment rates in the LI group indicate that liaison intervention is effective. The assessment implementation rates were $61.4 \%$ in the LI group and $52.1 \%$ in the non-LI group after 1 year and $54.3 \%$ in the LI group and $63.4 \%$ in the non-LI group after 2 years, and both groups showed extremely higher assessment implementation rates in the present study than in previous reports [20, 22]. In line with the higher osteoporosis treatment rates, extremely high bone density assessment rates were maintained because patients and physicians were extremely aware of the activity of the liaisons. These findings indicate that liaison intervention was effective in patients with fragility fractures.

Tracking investigations in Western Australia [26] conducted FLS interventions over 12 months in patients with fragility fractures who were aged over 50 years and found that refracturing decreased after 1 year in the intervention group compared with the control group; after adjusting for age and sex, participants with FLS intervention had significantly higher possibilities of implementing appropriate fracture risk assessments and osteoporosis treatments and significantly reduced fall rates compared with controls. The fall rates in the present study after 3 months were $3.0 \%$ in the LI group and $10.8 \%$ in the non-LI group; although no significant differences were observed, the fall rate was slightly lower in the LI group. Therefore, it is possible that FLS intervention might have preventative 
effects against falling in the short-term range. However, fall rates gradually increased after 3 months, and approximately one-third of patients in both groups experienced at least one fall 2 years after registration. There were no significant differences in the fall rate between the groups after 6 months or after 1 year. The higher osteoporosis treatment rate in the LI group might have contributed to the lower re-fracturing rate in the LI group after 1 year. Further investigations are needed to confirm the present findings.

Strengths of the present study are as follows: it was a randomized controlled trial and was conducted under clinical conditions with a 2-year tracking investigation, rather than a short-term study. Most of the previous studies that investigated the effects of liaison intervention were cluster-controlled trials or historical control trials, and to the best of our knowledge, the present study is the first randomized controlled trial to investigate the effects of liaison intervention.

However, this study has several limitations. First, this study was conducted by dividing patients into two groups at the same facility in an open trial, and the sample size was small; in addition, this study might have potential biases (Hawthorne effect). Most previous studies that showed the significant effects of coordinator intervention were cluster-controlled trials or historical control trials, and the large-scale randomized control trials [25] showed no significant differences in the treatment rates. We had considered that there are limitations to assessing the effects of coordinator intervention in randomized trials at the same facility. In addition, if the study period was extended or the number of subjects increased, we believe that this would have been a limiting factor for randomized controlled trials in the same facility.

Secondly, the type of osteoporosis medication administered was not considered in this study, and whether patients were taking supplements, such as vitamin D or calcium, was not analyzed. Future cluster randomized trials with a larger number of patients are needed to classify these issues.

Third, this study did not consider the effects of confounding variables on the present findings.

\section{Conclusions}

In this study, we conducted the first multicenter, randomized controlled trial in Japan with a follow-up period of 2 years and found that liaison intervention improved the bone mineral density assessment implementation rate, the osteoporosis treatment initiation rate, and the treatment persistence rates. Although these rates were high in both the LI and non-LI groups, significant differences between the two groups were observed in the early stages after the fracture. The present findings suggest that liaison intervention is a potential treatment option for physicians involved in fracture and osteoporosis treatment to assess osteoporosis and the commencement/continuation of osteoporosis drug treatments.

Acknowledgments We would like to express our gratitude to liaisons and staff at the seven hospitals and Dr. Noboru Yamagata who cooperated with this study. We are extremely thankful for the help with statistical analyses provided by Dr. Hisashi Noma (M.P.H, Ph.D.; assistant Professor) at the Department of Statistical Data Science at the Institute of Statistical Mathematics. The authors would also like to express sincere thanks to Ms. Ryoko Ikehara for her secretarial help.

Funding The present study was supported by the Ministry of Education, Culture, Sports, Science and Technology Grant-in-Aid for Scientific Research (16K10821) and funding from Tottori University.

Data availability The datasets generated and analyzed during the current study are not publicly available because they were part of patients' records but are available as a de-identified data sheet from the corresponding author upon reasonable request.

\section{Compliance with ethical standards}

Conflicts of interest $\mathrm{MO}, \mathrm{RO}, \mathrm{YS}, \mathrm{KT}, \mathrm{TN}, \mathrm{YM}$, and TO declare that they have no conflict of interest. HN received lecture fees or grants outside the submitted work from Asahi Kasei Pharma Corp.; Astellas Pharma Inc.; Chugai Pharmaceutical Co., Ltd.; Daiichi Sankyo Co., Ltd.; Eisai Co., Ltd.; Pfizer Inc.; Taisho Toyama Pharmaceutical Co., Ltd.; Teijin Pharma Co., Ltd; Takeda Pharmaceutical Co., Ltd.; Kaken Pharmaceutical Co., Ltd.; Shionogi \& Co., Ltd.; Nippon Zoki Pharmaceutical Co., Ltd.; Eli Lilly Japan Co., Ltd.; Hisamitsu Pharmaceutical Co., Ltd. MSD KK, and HH received lecture fees or grants outside the submitted work from Asahi Kasei Pharma Corp.; Astellas Pharma Inc.; Astellas Amgen Inc.; Chugai Pharmaceutical Co., Ltd.; Daiichi Sankyo Co., Ltd.; Eisai Co., Ltd.; Eli Lilly Japan Co., Ltd.; Mitsubishi Tanabe Pharma Corp.; Ono Pharmaceutical Co., Ltd.; Pfizer Inc.; Taisho Toyama Pharmaceutical Co., Ltd.; Teijin Pharma Co., Ltd; and UCB Japan Co., Ltd.

Ethics approval The study was approved by the ethics research committee at the Faculty of Medicine, Tottori University (No 2631) or each ethics committee of the sites that participated in the study.

Consent to participate Written informed consent was obtained from all patients before participating in this study.

Consent for publication Written consent for participation and consent for publication were obtained from all patients at the time of study enrollment.

Open Access This article is licensed under a Creative Commons Attribution-NonCommercial 4.0 International License, which permits any non-commercial use, sharing, adaptation, distribution and reproduction in any medium or format, as long as you give appropriate credit to the original author(s) and the source, provide a link to the Creative Commons licence, and indicate if changes were made. The images or other third party material in this article are included in the article's Creative Commons licence, unless indicated otherwise in a credit line to the material. If material is not included in the article's Creative Commons licence and your intended use is not permitted by statutory regulation or exceeds the permitted use, you will need to obtain permission directly from the copyright holder. To view a copy of this licence, visit http:// creativecommons.org/licenses/by-nc/4.0/. 


\section{References}

1. (2001) NIH Consensus Development Panel on osteoporosis prevention, diagnosis, and therapy. JAMA 285:785-795. https://doi. org/10.1001/jama.285.6.785

2. Klotzbuecher CM, Ross PD, Landsman PB, Abbott TA 3rd, Berger M (2000) Patients with prior fractures have an increased risk of future fractures: a summary of the literature and statistical synthesis. J Bone Miner Res 15:721-739. https://doi.org/10.1007/s11657-013-0136-1

3. Sakamoto K, Nakamura T, Hagino H, Endo N, Mori S, Muto Y, Harada A, Nakano T, Yamamoto S, Kushida K, Tomita K, Yoshimura M, Yamamoto H (2006) Report on the Japanese Orthopaedic Association's 3-year project observing hip fractures at fixed-point hospitals. J Orthop Sci 11:127-134. https://doi.org/ 10.1007/s00776-005-0998-1

4. Kado DM, Duong T, Stone KL, Ensrud KE, Nevitt MC, Greendale GA, Cummings SR (2003) Incident vertebral fractures and mortality in older women: a prospective study. Osteoporos Int 14:589594. https://doi.org/10.1007/s00198-003-1412-5

5. Fukui N, Watanabe Y, Nakano T, Sawaguchi T, Matsushita T (2012) Predictors for ambulatory ability and the change in ADL after hip fracture in patients with different levels of mobility before injury: a 1-year prospective cohort study. J Orthop Trauma 26:163171. https://doi.org/10.1097/BOT.0b013e31821e1261

6. Beaupre LA, Jones CA, Johnston DW, Wilson DM, Majumdar SR (2012) Recovery of function following a hip fracture in geriatric ambulatory persons living in nursing homes: prospective cohort study. J Am Geriatr Soc 60:1268-1273. https://doi.org/10.1111/j. 1532-5415.2012.04033.x

7. Roerholt C, Eiken P, Abrahamsen B (2009) Initiation of antiosteoporotic therapy in patients with recent fractures: a nationwide analysis of prescription rates and persistence. Osteoporos Int 20: 299-307. https://doi.org/10.1007/s00198-008-0651-x

8. Hagino H, Sawaguchi T, Endo N, Ito Y, Nakano T, Watanabe Y (2012) The risk of a second hip fracture in patients after their first hip fracture. Calcif Tissue Int 90:14-21. https://doi.org/10.1007/ s00223-011-9545-6

9. Tamaki J, Fujimori K, Ikehara S, Kamiya K, Nakatoh S, Okimoto N, Ogawa S, Ishii S, Iki M, Working Group of Japan Osteoporosis Foundation (2019) Estimates of hip fracture incidence in Japan using the National Health Insurance Claim Database in 20122015. Osteoporos Int 30:975-983. https://doi.org/10.1007/ s00198-019-04844-8

10. Marsh D, Akesson K, Beaton DE, Bogoch ER, Boonen S, Brandi ML, McLellan AR, Mitchell PJ, Sale JE, Wahl DA (2011) Coordinator-based systems for secondary prevention in fragility fracture patients. Osteoporos Int 22:2051-2065. https://doi.org/10. 1007/s00198-011-1642-x

11. McLellan AR, Wolowacz SE, Zimovetz EA, Beard SM, Lock S, McCrink L, Adekunle F, Roberts D (2011) Fracture liaison services for the evaluation and management of patients with osteoporotic fracture: a cost-effectiveness evaluation based on data collected over 8 years of service provision. Osteoporos Int 22:2083-2098. https://doi.org/10.1007/s00198-011-1534-0

12. Moriwaki K, Noto S (2017) Economic evaluation of osteoporosis liaison service for secondary fracture prevention in postmenopausal osteoporosis patients with previous hip fracture in Japan. Osteoporos Int 28: 621-632. https://doi.org/10.1007/s00198-016-3777-2

13. Hagino H, Wada T (2019) Osteoporosis liaison service in Japan. Osteoporos Sarcopenia 5:65-68. https://doi.org/10.1016/j.afos. 2019.09.003
14. (1987) The prevention of falls in later life. A report of the Kellogg International Work Group on the prevention of falls by the elderly. Dan Med Bull 34 Suppl 4:1-24

15. Mitchell PJ (2011) Fracture liaison services: the UK experience. Osteoporos Int 22(Suppl 3):487-494. https://doi.org/10.1007/ s00198-011-1702-2

16. Gardner MJ, Brophy RH, Demetrakopoulos D, Koob J, Hong R, Rana A, Lin JT, Lane JM (2005) Interventions to improve osteoporosis treatment following hip fracture. A prospective, randomized trial. J Bone Joint Surg Am 87:3-7. https://doi.org/10.2106/JBJS.D.02289

17. Wright NC, Looker AC, Saag KG, Curtis JR, Delzell ES, Randall S, Dawson-Hughes B (2014) The recent prevalence of osteoporosis and low bone mass in the United States based on bone mineral density at the femoral neck or lumbar spine. J Bone Miner Res 29:2520-2526. https://doi.org/10.1002/jbmr.2269

18. Huntjens KM, van Geel TA, van den Bergh JP, van Helden S, Willems P, Winkens B, Eisman JA, Geusens PP, Brink PR (2014) Fracture liaison service: impact on subsequent nonvertebral fracture incidence and mortality. J Bone Joint Surg Am 96:e29. https://doi.org/10.2106/JBJS.L.00223

19. Dell R, Greene D (2010) Is osteoporosis disease management cost effective? Curr Osteoporos Rep 8:49-55. https://doi.org/10.1007/ s11914-010-0009-0

20. Feldstein A, Elmer PJ, Smith DH, Herson M, Orwoll E, Chen C, Aickin M, Swain MC (2006) Electronic medical record reminder improves osteoporosis management after a fracture: a randomized, controlled trial. J Am Geriatr Soc 54:450-457. https://doi.org/10. 1111/j.1532-5415.2005.00618.x

21. Gonzalez-Quevedo D, Bautista-Enrique D, Perez-Del-Rio V, Bravo-Bardaji M, Garcia-de-Quevedo D, Tamimi I (2020) Fracture liaison service and mortality in elderly hip fracture patients: a prospective cohort study. Osteoporos Int 31:77-84. https://doi.org/10.1007/s00198-019-05153-w

22. Axelsson KF, Jacobsson R, Lund D, Lorentzon M (2016) Effectiveness of a minimal resource fracture liaison service. Osteoporos Int 27:3165-3175. https://doi.org/10.1007/s00198016-3643-2

23. Morberg BM, Malling AS, Jensen BR, Gredal O, Wermuth L, Bech P (2018) The Hawthorne effect as a pre-placebo expectation in Parkinsons disease patients participating in a randomized placebocontrolled clinical study. Nord J Psychiatry 72:442-446. https://doi. org $/ 10.1080 / 08039488.2018 .1468480$

24. Abdulraheem SBL (2018) Hawthorne effect reporting in orthodontic randomized controlled trials: truth or myth? Blessing or curse? Eur J Orthod 40:475-479. https://doi.org/10.1093/ejo/cjx089

25. Solomon DH, Katz JN, Finkelstein JS, Polinski JM, Stedman M, Brookhart MA, Arnold M, Gauthier S, Avorn J (2007) Osteoporosis improvement: a large-scale randomized controlled trial of patient and primary care physician education. J Bone Miner Res 22:1808-1815. https://doi.org/10.1359/jbmr.070717

26. Inderjeeth CA, Raymond WD, Briggs AM, Geelhoed E, Oldham D, Mountain D (2018) Implementation of the Western Australian Osteoporosis Model of Care: a fracture liaison service utilising emergency department information systems to identify patients with fragility fracture to improve current practice and reduce refracture rates: a 12-month analysis. Osteoporos Int 29:1759-1770. https://doi.org/10.1007/s00198-018-4526-5

Publisher's note Springer Nature remains neutral with regard to jurisdictional claims in published maps and institutional affiliations. 